\title{
The impact of the reinforcement percentage on the stress-strain state of the bending steel fiber reinforced concrete elements
}

\author{
Oleksandr Andriichuk ${ }^{1, *}$, Volodymyr Babich ${ }^{2}$, Ivan Yasyuk $^{1}$, and Serhii Uzhehov ${ }^{1}$ \\ ${ }^{1}$ Lutsk National Technical University, 43018 Lutsk, Lvivska St. 75, Ukraine \\ ${ }^{2}$ National University of Water Management and Nature Resources Use, 33000 Rivne, Soborna St. 1, \\ Ukraine
}

\begin{abstract}
The use of concrete with traditional reinforcement pose the problem of finding ways to increase the crack resistance, impact strength, frost resistance and other characteristics, which in turn depend on the strength properties of the material. One of the solution is the use of dispersion reinforced concrete - steel fiber concrete (with short steel fibers of $30-50 \mathrm{~mm}$ ). The combination of rigid fibers with significant strength reserves and the concrete could increase the crack resistance of the matrix and other strength characteristics. The paper presents the results of theoretical studies of the reinforcement percentage effect on the stressstrain state of the rectangular cross-section elements of steel fiber reinforced concrete (SFRC) in bending from at repeated loads with levels of application $\eta=0.5$ and $\eta=0.7$. It is found that the limit deformations of the SFRC cross-section in bending is recommended to calculate using a polynomial function, which gives the greatest convergence between the true values and approximations in comparison with the logarithmic and exponential functions.
\end{abstract}

\section{Introduction}

During the authors' previous studies, it was found that steel fiber reinforced concrete (SFRC) as an effective material should be used for the production of gutter drainage systems [1-2]. The use of SFRC gutters instead of standard reinforced concrete gutters allows to completely avoid the use of rebar; reduce the consumption of materials of construction, and as a consequence, their weight; reduce labor costs for production of structures and significantly increase the durability of constructions. These studies were conducted in the building laboratory of Lutsk NTU. It should be noted that the effectiveness of the use of dispersion-reinforced concrete is confirmed by the results of studies and other authors [3-5].

In recent years, the study of dispersion reinforced concrete and structures based on them, working under the influence of repeated static and dynamic loads, has gained significant relevance. Leaders in these studies are the Russian Federation $[6,7]$ and Israel

\footnotetext{
* Corresponding author: aleklutsk@gmail.com
} 
[8] because high-strength fiber reinforced concrete hold a great promise in the development of modern protective structures for defense purposes. It should be noted that at present, in other countries, researches are carried out to increase the strength properties of structural elements by using steel fibers for their dispersed reinforcement [9-12].

To obtain steel fiber reinforced concrete as a dispersed reinforcement of experimental gutters, it is used a wave fiber with a diameter of $0.8 \mathrm{~mm}$ and length of $50 \mathrm{~mm}$ (Fig. 1).

The geometric dimensions of the gutters were as follows: wall thickness $t=40 \mathrm{~mm}$; an inner diameter $d_{\text {in }}=300 \mathrm{~mm}$; outer diameter $d_{\text {out }}=380 \mathrm{~mm}$; length of the elements $l=300 \mathrm{~mm}$. General view of SFRC gutters is shown in Fig. 1.

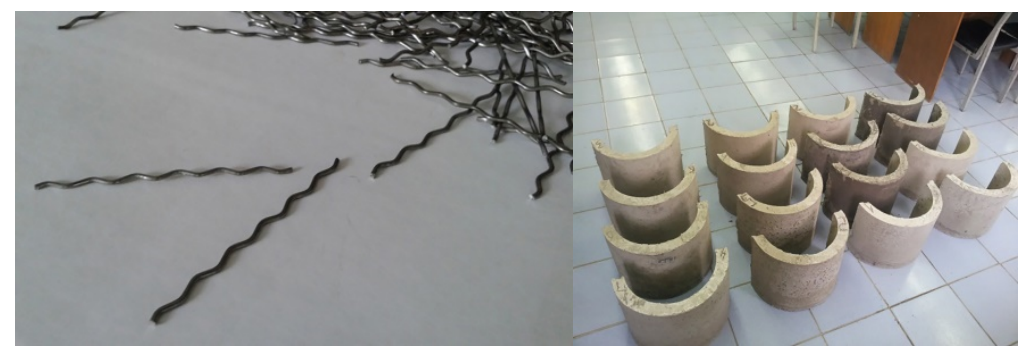

Fig. 1. General view of steel anchor fibers for the production of steel fiber reinforced concrete.

During the experimental studies [2], it was determined the actual stress-strain state of SFRC gutters with reinforcement coefficients $\mu=1, \mu=2$ and $\mu=3$ from the effect of repeated loads on them with application levels $\eta=0.5$ and $\eta=0.7$ (within ten cycles). At the 11th cycle the load increased to destructive for determining the bearing capacity of the elements. The main factors that are determined the stress-strain state of structures should be: linear dimensions of the structure and its cross sections that deform; type of applied load; modulus of elasticity of material.

The moments of resistance $(W)$ of the rectangular cross-section for three different percent of reinforcement with a variation of $1 \%(\mu=1,, \mu=2$ and $\mu=3)$ of the SFRC gutters are characterized by the dependence:

$$
W=\frac{b \times h^{2}}{6}
$$

$b$ - width of section; $h$ - height (thickness) of section;

The resulting stresses in concrete and SFRC are characterized by the expression:

$$
\sigma=M / W
$$

Due to the fact that the geometry of the gutters cross-sections $\left(\frac{b \times h^{2}}{6}\right)$ at different percentages of reinforcement ( $\mu=1, \mu=2$ and $\mu=3$ ) is constant, the stress-strain state of the cross section can be estimated on the basis of the limiting moment that the research elements are takes at different percentages of reinforcement and, respectively, the emerging limit stresses in concrete and SFRC $(\sigma)$.

On the basis of experimentally obtained data of limit stresses in the concrete section of the gutter $\left(\sigma_{c}\right)$ and SFRC cross-sections $\left(\sigma_{s f}\right)$, depending on the percentage of reinforcement $(\mu)$, it is advisable to describe the functional relationship between them $f(\mu)$ given by the expression: 


$$
\sigma_{s f}=f(\mu) \cdot \sigma_{c}
$$

It should be noted that at $\mu=0$ (concrete element) the function $f(\mu)=1$.

Then the dependence of the limit stresses in the SFRC section $\left(\sigma_{s f}\right)$ on the experimentally determined limit stresses in the base concrete section $\left(\sigma_{c}\right)$ can be found using the determined function, which depends on the percentage of reinforcement $f(\mu)$.

Experimental results of testing samples according to the experimental plan [2] are presented in Table 1.

Table 1. Bearing capacity of steel fiber reinforced concrete cross-sections of gutters.

\begin{tabular}{|c|c|c|c|c|c|}
\hline \multirow{2}{*}{$\begin{array}{c}\text { The level of applied } \\
\text { repeated loads }\end{array}$} & \multirow{2}{*}{ Limit stress } & \multicolumn{4}{|c|}{ The percentage of reinforcement, \% } \\
\cline { 4 - 6 } & & $\mu=0$ & $\mu=1$ & $\mu=2$ & $\mu=3$ \\
\hline$\eta=0.5$ & $\sigma, M P a$ & 15.88 & 19.40 & 27.72 & 30.64 \\
\cline { 4 - 6 } & & & 17.03 & 27.72 & 29.69 \\
\hline
\end{tabular}

To determine the intermediate values of the stress limits for other percentages of reinforcement, we apply the least squares method, which is one of the methods of regression analysis, that is used for statistical evaluation of the regression model parameters from empirical data.

According to this method, the parameters of the model must correspond to a regression equation which provides the smallest value of the sum of the squares of deviations of empirical data from those calculated by the regression equation. Thus, of two different approximations of the same empirical function, which is given in the form of a table, the best is the one for which the sum of the squares of the deviation has the smallest value. A generalization of the conditions for the application of the least squares method is formulated in the Gauss-Markov theorem [13].

Dependences of limit stress on the reinforcement ratio $(\sigma-\mu)$ for cross-section of SFRC gutters with the intermediate values are presented in Fig. 2, with $\eta=0.5$ and $\eta=0.7$.

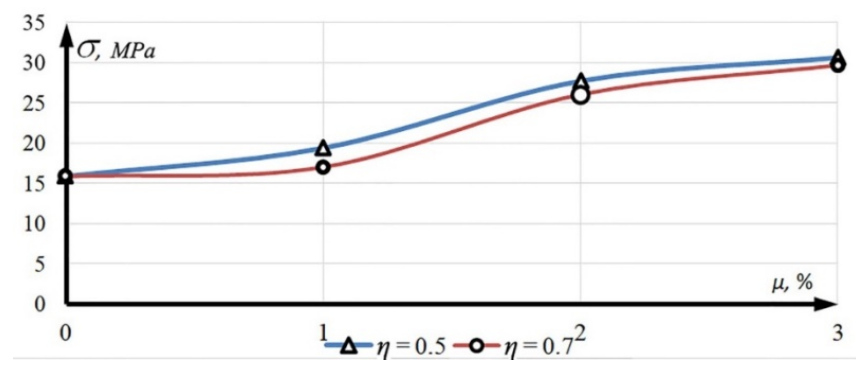

Fig. 2. Dependences of limit stress on the reinforcement ratio of cross-section of SFRC gutters.

Let approximate the obtained curves at the load levels of SFRC gutters $\eta=0.5$ and $\eta=0.7$ using three functions (logarithmic $y=\log _{a} x$, exponential $y=e^{x}$ and polynomial $\left.y=c_{0}+c_{1} x+\ldots+c_{n} x^{n}\right)$ and build the corresponding trend lines. At the level of repeated loads $\eta=0.5$ the trend lines for the approximated curve are shown in Fig. 3 (a, b, c), and at the load level $\eta=0.7$ are shown in Fig. 4 (a, b, c). 


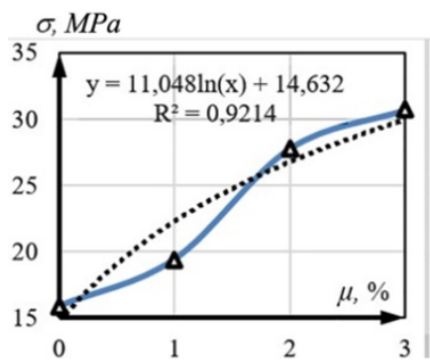

a)

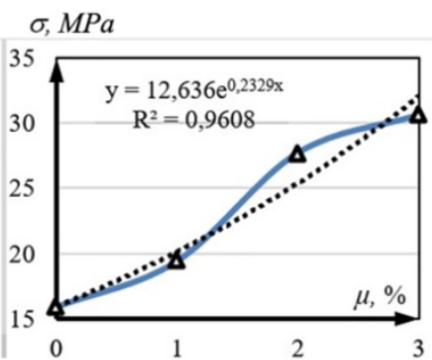

b)

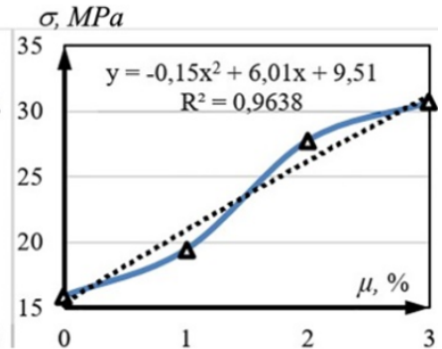

c)

Fig. 3. Obtained by approximating the trend line at $\eta=0.5$ using (a) the logarithmic function; (b) the exponential function; (c) the polynomial function.

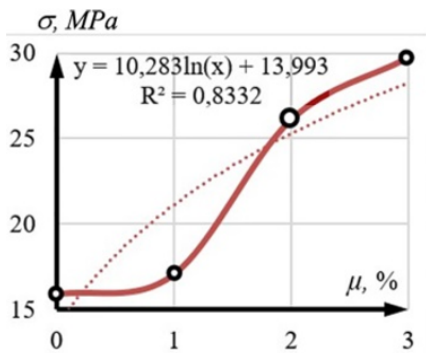

a)

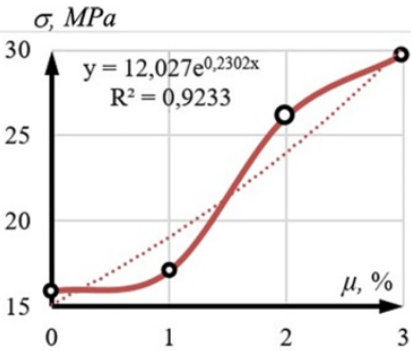

b)

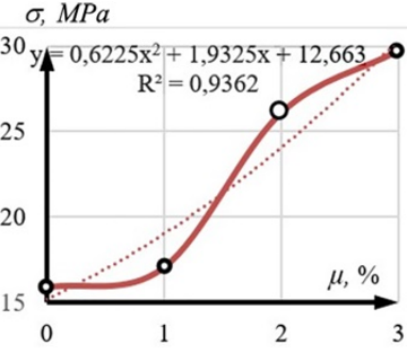

c)

Fig.4. Obtained by approximating the trend line at $\eta=0.7$ using (a) the logarithmic function;

(b) the exponential function; (c) the polynomial function.

Let also determine the deviation - correlation coefficients $\left(R^{2}\right)$ for each of the functions obtained by approximation at $\eta=0.5$ and $\eta=0.7$.

At the approximation of data using the logarithmic functions and given that $f(\mu)=\sigma_{s f} / \sigma_{c}$ (according to (3)), limit strain of SFRC sections are:

at $\eta=0.5 \quad \sigma_{s f}=(11.048 \ln (\mu)+14.632) \cdot \sigma_{c}$

at $\eta=0.7$

$$
\sigma_{s f}=(11.048 \ln (\mu)+14.632) \cdot \sigma_{c}
$$

At the approximation of data using the exponential function and given (3), limit strain of SFRC sections are:

$$
\begin{array}{ll}
\text { at } \eta=0.5 & \sigma_{s f}=12.636 e^{0.2329 \mu} \cdot \sigma_{c} \\
\text { at } \eta=0.7 & \sigma_{s f}=12.027 e^{0.2302 \mu} \cdot \sigma_{c}
\end{array}
$$

At the approximation of data using the polynomial function and given (3), limit strain of SFRC sections are:

$$
\begin{array}{ll}
\text { at } \eta=0.5 & \sigma_{s f}=\left(-0.15 \mu^{2}+6.01 \mu+9.51\right) \cdot \sigma_{c} \\
\text { at } \eta=0.7 & \sigma_{s f}=\left(0.6225 \mu^{2}+1.9325 \mu+12.663\right) \cdot \sigma_{c}
\end{array}
$$

The correlation coefficients at the level $\eta=0.5$ are determined as follows: $R_{\log }^{2}=0.9214 ; R_{\text {exp }}^{2}=0.9608 ; R_{p o l}^{2}=0.9638$. The correlation coefficients at the level $\eta=0.7$ are determined as follows: $R_{\log }^{2}=0.8332 ; R_{\text {exp }}^{2}=0.9233 ; R_{p o l}^{2}=0.9362$. 


\section{Conclusions}

The studies that are presented in the article provide the possibility of determining the limit stresses in the cross section of the steel fiber reinforced concrete element from its reinforcement percentage. This requires only the presence of the strength characteristics of the concrete section.

After analyzing the obtained correlation coefficients, it can be argued that the greatest convergence between the true values and approximations is in the application of the polynomial function in accordance with the equations $8-9$ (at $\eta=0.5$ and at $\eta=0.7$ ).

It is found that the limit deformation of the cross-section of SFRC in bending is recommended to calculate using a polynomial function, which gives the greatest convergence $(95 \%)$ among the true values and approximations in comparison with the logarithmic and exponential functions.

\section{References}

1. E.M. Babych, O.V. Andriichuk. Strength of elements with annular cross sections made of steel-fiber-reinforced concrete under one-time loads. Materials Science, New York, 52, 509-513 (2017)

2. O. Andriichuk, V. Babich, I. Yasyuk, S. Uzhehov. MATEC Web of Conferences, 116, 02001, 1-9 (2017)

3. L. Dvorkin, O. Dvorkin, Y. Ribakov. A method for optimal design of steel fiber reinforced concrete composition. Materials and Design (2011)

4. R. Kinash, V. Bilozir. Deformational calculation method of bearing capability of fiberconcrete steel bending elements. Czasopismo Techniczne (2015)

5. R. Shmyh, V. Bilozir, A. Vysochenko, V. Bilozir. Carrying capacity of bending concrete elements reinforced by fibro and stripes taken from used PET bottles. International Scientific and Practical Conference World science (2018)

6. D.A. Lamzin. High-speed deformation and destruction of fine concrete. Thesis. (2014)

7. L.A. Avetisyan. Strength and deformability of compressed reinforced concrete elements under dynamic loading under fire conditions. Thesis. (2015)

8. S.U. Baloch. Steel fiber reinforced concrete. Science Technology and Development 24, 21-32 (2015)

9. S.V. Klyuyev, A.V. Klyuyev, R.V. Lesovik and A.V. Netrebenko. World Applied Sciences Journal 24: 1280-1285 (2013)

10. A. Winkler, C. Edvardsen, T. Kasper. Examples of bridge, tunnel lining and foundation design with steel-fibre-reinforced concrete. Fibre-reinforced concrete from design to structural applications. 310, 451-460 (2017)

11. J. Ming, J. Shi, W. Sun. Effect of mill scale on the long-term corrosion resistance of a low-alloy reinforcing steel in concrete subjected to chloride solution. Construction and building materials. 163, 508-517 (2018)

12. J. Yuan and B.A. Graybeal. Bond behavior of reinforcing steel in ultra-high performance concrete. Federal highway administration (2014)

13. H. James, H. Stock, W. Mark. Regression with a single regressor: hypothesis tests and confidence intervals. Introduction to Econometrics, 3, Addison-Wesley, 163-164. 785 (2011) 